\title{
A Novel Particle/Photon Detector Based on a Superconducting Proximity Array of Nanodots
}

\author{
Daniele Di Gioacchino $^{1}$ - Nicola Poccia ${ }^{2,3}$ - Martijn Lankhorst ${ }^{2}$ - Claudio Gatti ${ }^{1}$. \\ Bruno Buonomo $^{1}$ - Luca Foggetta ${ }^{1}$. Augusto Marcelli ${ }^{1,4}$ • Hans Hilgenkamp ${ }^{2}$
}

Received: 16 June 2016 / Accepted: 6 August 2016 / Published online: 28 September 2016

(C) The Author(s) 2016. This article is published with open access at Springerlink.com

\begin{abstract}
The current frontiers in the investigation of highenergy particles demand for new detection methods. Higher sensitivity to low-energy deposition, high-energy resolution to identify events and improve the background rejection, and large detector masses have to be developed to detect even an individual particle that weakly interacts with ordinary matter. Here, we will describe the concept and the layout of a novel superconducting proximity array which show dynamic vortex Mott insulator to metal transitions, as an ultra-sensitive compact radiation-particle detector.
\end{abstract}

Keywords Proximity effects · Superconductivity · Josephson vortex · Vortex Mott transitions · Detector

\section{Introduction}

The physics and the recent observation of a dynamic vortex Mott transition in a superconducting proximity array

Nicola Poccia

n.poccia@utwente.nl

Daniele Di Gioacchino

daniele.digioacchino@lnf.infn.it

1 Istituto Nazionale di Fisica Nucleare, Laboratori Nazionali di Frascati, 00044 Frascati (RM), Italy

2 MESA Institute for Nanotechnology, University of Twente, P. O. Box 217, 7500AE Enschede, Netherlands

3 NEST Istituto Nanoscienze-CNR \& Scuola Normale Superiore, Pisa, Italy

4 RICMASS - Rome International Center for Materials Science Superstripes, Via dei Sabelli 119A, 00185 Roma, Italy
[1] together with the evidence of Shapiro steps measured at gigahertz [2] suggest the possibility to design a conceptually new particle/radiation detector. We propose here the layout and of a possible setup of a device. While it is well known that a superconductive device can be used as sensitive detector of various quantities that can be converted in a very small magnetic field signal, it is also know that a superconducting particle detector may exhibit an extremely high-energy resolution, actually proportional to $\sqrt{ } E$ that is particularly attractive because of the small value of the superconducting energy gap $(E \approx \mathrm{meV})$ to be compared with the typical energetic excitation in a semiconductor $(E \sim \mathrm{eV})$ and of a gas detector $(E \sim 25 \mathrm{eV})$. These detectors could be then suitable to detect solar neutrinos, WIMPS and other weakly interacting particles being the sensitivity of these devices proportional to a very small energy deposition [3].

A superconductive device can, in principle, detect a magnetic flux with sensitivity around $10^{-6} \Phi_{0}$ ( $\Phi_{0}=h / 2 \mathrm{e} \approx 2.06710^{-15}$ Weber) using a SQUID sensor. Such devices can identify particles and/or photons in the long wavelength, optical, and x-ray range using thermal (bolometric) or non-bolometric effects [4]. However, in general, for the low frequency regime up to microwave/IR wavelengths the photon energy is too small with respect to the noise floor and the response is mainly due to the large number of absorbed photons In the high frequency domain, from the visible to the x-ray domain, such detectors are sensitive enough to probe the signal due to the absorption of a single photon or a single particle [5] Nevertheless, at present, the energy resolution of the best ultrasensitive single photon superconducting bolometric detectors show, down to approximately $20 \mathrm{GHz}$, an energy resolution near the ideal values due to the intrinsic thermal fluctuation noise [6].

The bolometric effect, which is behind these devices, is based to the temperature rise induced by particles/incident 
radiation on a thin superconductor film, measuring the large temperature change of the DC resistance or inductance of the film The mechanism implies that the film remains in a thermodynamic equilibrium throughout the duration of the modulation due to the incident particle/photon illumination [7]. An example of existing high-energy particle bolometric device is the superconducting transition-edge sensor (TES) that works on the cusp of the superconducting transition where even a small change of the temperature will cause an abrupt change in the resistance [8].

Non-bolometric effects are associated to the occurrence of nonequilibrium phenomena in superconducting thin film [4] or Josephson junction network [9, 10]. A device based on a non-bolometric effect may lead to the detection even of a single photon, for example considering the radiation frequency in the internal Josephson oscillation range, i.e., from megahertz to gigahertz, up to the terahertz domain synchronization effects know as Shapiro steps occur. In this case we detect a jump of the current when the average voltage is an integer multiple of the AC frequency divided by the Josephson constant $2 \mathrm{e} / \mathrm{h}=483597011 \mathrm{GHz} / \mathrm{V}$ [1-12] Another non-bolometric device could be made with a "superconducting nanowire" $100 \mathrm{~nm}$ wide. This simple device may operate at a temperature well below the superconducting transition temperature of the corresponding film when the sample is biased just below the critical current. When a "radiation" strikes this wire, a local resistive hotspot forms, inducing a perturbation of the current distribution so that a fast voltage-pulse can be measured [8] In this process vortices have a role in the detection because after the hotspot the superconducting state disappears, being associated to the appearance of a vortex-antivortex pair [13] It is evident that it needs to control the superconducting properties at nanoscale [14-16].

\section{The Superconducting Proximity Array as a Radiation Detection}

To analyze the possibility of using a proximity superconducting array [1] as a new multipurpose radiation detector, we briefly summarize here some characteristics of the device we assembled It was manufactured on a silicon/silicon oxide substrate where a metallic gold template with four contacts has been grown. The size of the array is $80 \mu \mathrm{m} \times 80 \mu \mathrm{m}$. On this "template" an array of $300 \times 300=90,000 \mathrm{Nb}$ superconducting islands was realized. As shown in ref. [1] the device has a period of $270 \mathrm{~nm}$, the island diameter is $220 \mathrm{~nm}$ and, considering an island thickness of $45 \mathrm{~nm}$ the separation is only $47 \mathrm{~nm}$.

After the application of a magnetic field, Josephson vortices are induced and localized among superconducting islands because of the weaker superconductivity proximity effect of the superconducting-normal metalsuperconducting (SNS) junctions. The vortices will distribute in a regular way among $\mathrm{Nb}$ nanodots as a function of the magnetic field. These Josephson vortices can generate different patterns [1]. The configuration of the vortex lattices is a function of $f$, where $f=B / B_{0}$, with $B$ the applied magnetic field and $B_{0}=\Phi_{0} / a^{2}=28.6 \mathrm{mT}$ [1]. Here, $a$ is the distance among $\mathrm{Nb}$ nanodots defined by the geometrical pattern of the device. In particular, the system will show the minimum of the differential electrical resistance, $\mathrm{dV} / \mathrm{dI}$, at different stable Josephson vortex lattices determined by the parameter $f=B / B_{0}$. Moreover, in addition to the minimum values, the differential resistance shows also a maximum at a fixed magnetic field depending by the ac current level. We have to underline here that looking at the $\mathrm{V} / \mathrm{I}$ characteristics, the working mechanism is not a depinning phenomenon [1].
Table 1 The main parameters of the DAФNE-Beam Test Facility (BTF)

\begin{tabular}{|c|c|c|c|c|}
\hline Parameter & \multicolumn{4}{|c|}{ Values } \\
\hline Maximum average flux & \multicolumn{4}{|c|}{$3.12510^{10}$ particles $/ \mathrm{s}$} \\
\hline Spot size & \multicolumn{4}{|c|}{$\begin{array}{l}1-25 \mathrm{~mm}(\mathrm{y}) \\
1-55 \mathrm{~mm}(\mathrm{x})\end{array}$} \\
\hline Divergence & \multicolumn{4}{|c|}{$1-2 \mathrm{mrad}$} \\
\hline & \multicolumn{2}{|c|}{ Parasitic mode } & \multicolumn{2}{|c|}{ Dedicated mode } \\
\hline Pulse duration & \multicolumn{2}{|r|}{$10 \mathrm{~ns}$} & \multicolumn{2}{|c|}{$\begin{array}{l}1.5-40 \mathrm{~ns} \\
\text { Selectable }\end{array}$} \\
\hline \multirow[t]{2}{*}{ Repetition rate } & \multicolumn{2}{|c|}{$\begin{array}{l}\text { Variable between } 10 \mathrm{~Hz} \text { and } 49 \mathrm{~Hz} \\
\text { Depending on DAFNE mode }\end{array}$} & \multicolumn{2}{|c|}{$\begin{array}{l}1-49 \mathrm{~Hz} \\
\text { Selectable }\end{array}$} \\
\hline & With target & Without target & With target & Without target \\
\hline Particle species & $\begin{array}{c}e^{*} \text { or } e^{-} \\
\text {Selectable by user }\end{array}$ & $\begin{array}{c}e^{*} \text { or } e^{-} \\
\text {Depending on DAFNE mode }\end{array}$ & \multicolumn{2}{|c|}{$\begin{array}{l}\mathrm{e}^{+} \text {or } \mathrm{e}^{-} \\
\text {Selectable }\end{array}$} \\
\hline Energy & $25-500 \mathrm{MeV}$ & $510 \mathrm{MeV}$ & $\begin{array}{l}25-700 \mathrm{MeV}\left(\mathrm{e}^{-}\right) \\
25-500 \mathrm{MeV}\left(\mathrm{e}^{+}\right)\end{array}$ & $\begin{array}{l}250-730 \mathrm{MeV}^{-\left(\mathrm{c}^{-}\right)} \\
250-530 \mathrm{MeV}\left(\mathrm{e}^{+}\right)\end{array}$ \\
\hline Energy spread & $1 \%$ at $500 \mathrm{MeV}$ & $0.5 \%$ & \multicolumn{2}{|c|}{$0.5 \%$} \\
\hline $\begin{array}{c}\text { Intensity } \\
\text { (particles/bunch) }\end{array}$ & $1-10^{5}$ & $10^{7}-1.510^{10}$ & $1-10^{5}$ & $3^{3}-310^{10}$ \\
\hline
\end{tabular}



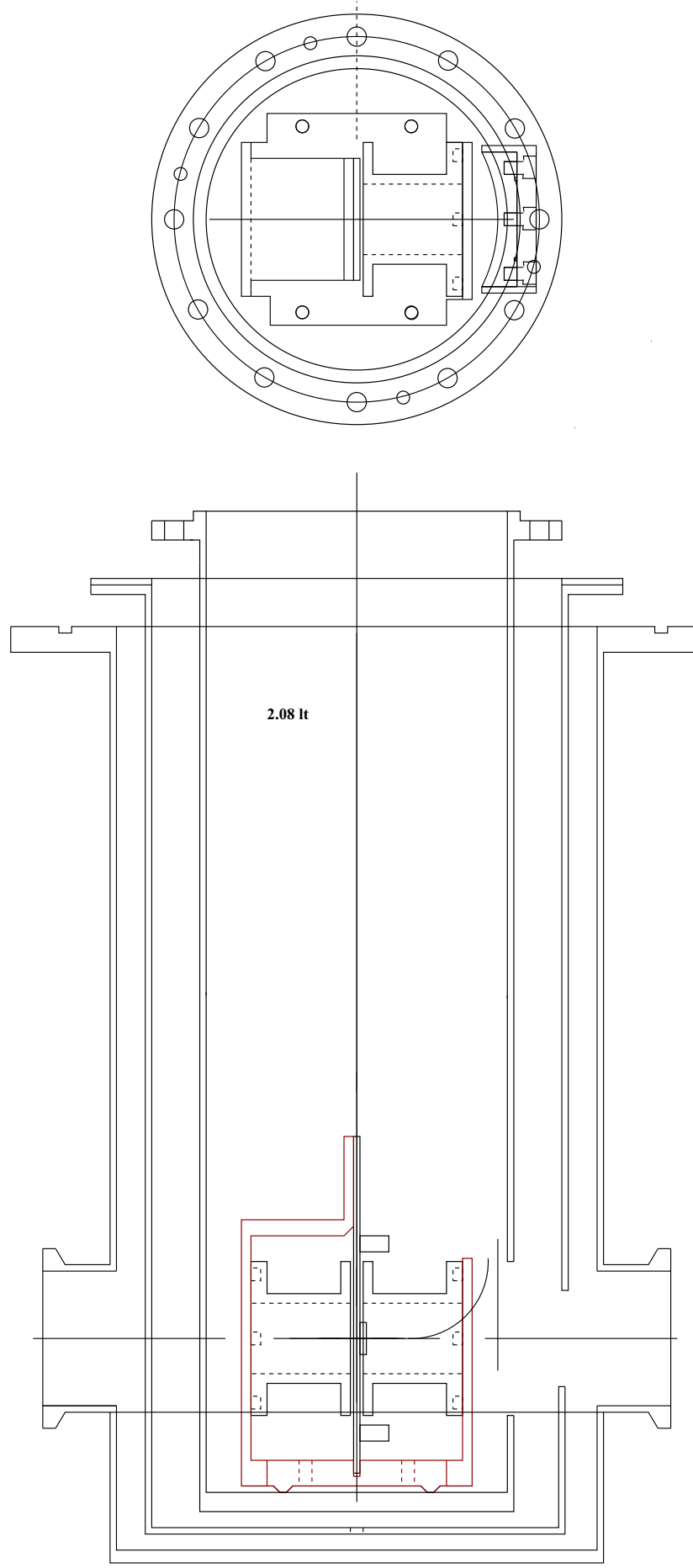

Fig. 1 The cryostat layout

The particle-radiation interaction time process in a superconducting proximity array can be expected in the femtosecond time scale and the energies involved in the $\mathrm{meV}$ regime. To determine if a superconducting proximity array with the above characteristics could be used as a novel generation radiation-particle detector, a prototype is currently under tests. Preliminary results are briefly described below.

\section{Experimental Setup and Future Tests of the New Radiation Detector}

In this section, we describe the experimental layout and the results of the preliminary tests of a device undergoing a radiation. The response of this prototype will be measured using charged particles produced by the DA $\Phi$ NE-Beam Test Facility (BTF) http://www.lnf.infn.it/acceleratori/btf/ of the LNF. The BTF is a beam transfer line that allows the diversion of the primary beams (electrons or positrons, $510 \mathrm{MeV}, 10 \mathrm{~ns}$ bunch length) produced by the high intensity LINAC, mainly with the purpose of testing, characterizing and calibrating particle detectors. The facility provide runtime tuneable electron and positron beams with a wide range of selection both in energy and in multiplicity. One possible BTF setup ("low multiplicity" one) is based on a secondary beam devoted to the stochastic production of single electrons/positrons, for detector calibration purposes, or for the extraction of the DA $\Phi$ NE LINAC electron/positron beam. In Table 1 are summarized its main beam characteristics.

To perform experiments at cryogenic temperatures, we assembled a liquid helium cryostat to accommodate the card with the superconducting devices, one of which is connected to the circuit lines for electrical tests. The original cryostat layout with all vacuum flanges is showed in the left of Fig. 1. On its top, we have the liquid nitrogen filling feedthrough of the internal thermal shield vessel, the liquid helium feedthrough that fills the sample vessel, the electrical feedthroughs used for the I-V measurements, the power supply of the electrical connections of the superconducting coil, temperature controls, and vacuum connections.

The new layout is shown in the right panel of Fig. 1. The cryostat bottom vessel hosts the electronic card with the proper windows to match the illumination of the radiation provided by the BTF source. In the next, in Fig. 2 are shown the different components that compose the system:

a. A plastic PEEK sample-holder with the superconducting device electronic card and the NbTi superconducting coil. This latter components will produce a magnetic field from 0 to $100 \mathrm{mT}$ and sets a Josephon vortex configuration in the device (panels a-c);

b. The window flanges are targets of the device relatively to the position of the incoming electron bunch. Moreover, this layout takes into account the possibility to insert from the top flange UV-visible-IR fibers for radiation tests.

We performed a preliminary simulation with GEANT4 program https://geant4.web.cern.ch/geant4/, to evaluate at $500 \mathrm{MeV}$ the e-beam divergence when it interacts with the window passive material in the cryostat. The parameters we considered are as follows: (i) $200 \mu \mathrm{m}$ of aluminum 7075 of the cryostat external window, (ii) $100 \mu \mathrm{m}$ of aluminized 
Fig. 2 a, b Two side views of the sample holder and $\mathbf{c}$ the sample holder from the top
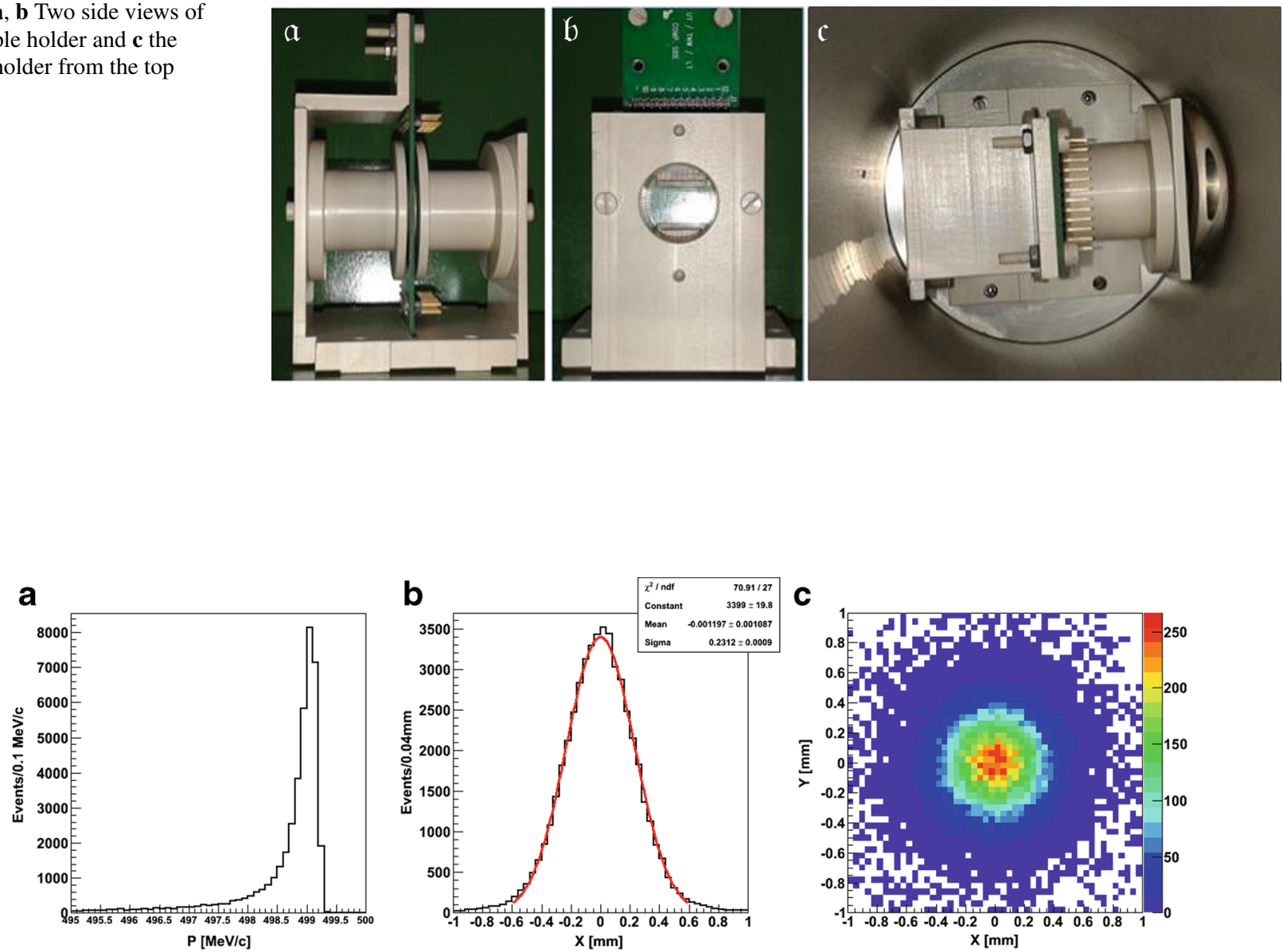

Fig. 3 Electron simulation results with GEANT4: a the bunch energy Bremsstrahlung, $\mathbf{b}$ the bunch Gaussian distribution, and $\mathbf{c}$ the bunch $x-y$ distribution
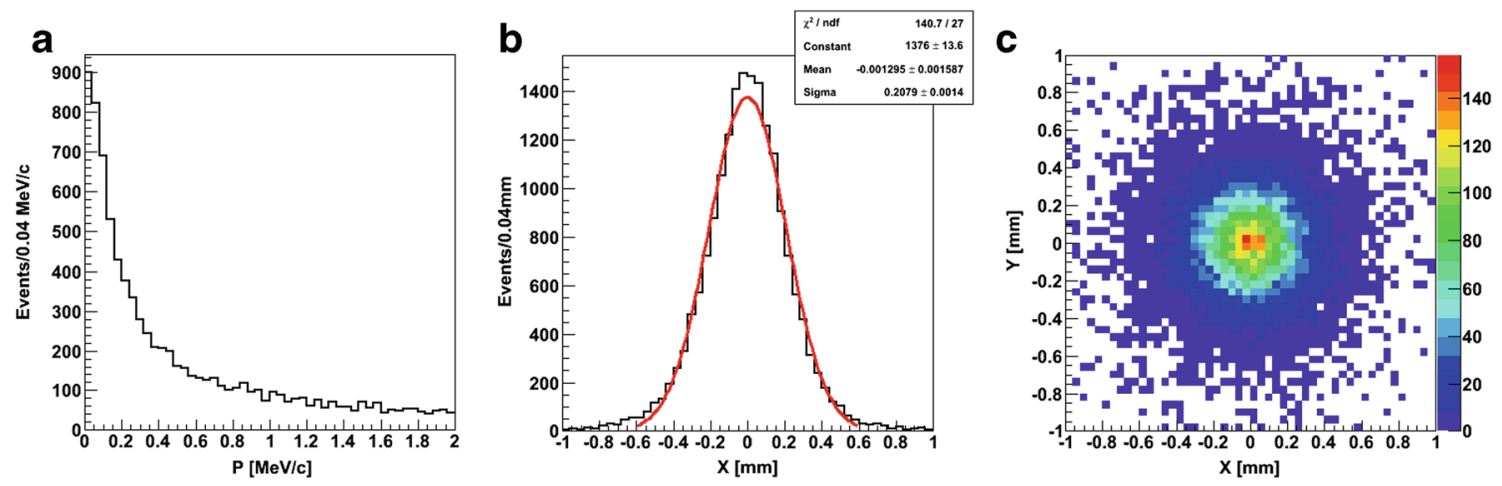

Fig. 4 Photon simulation results with GEANT4: a the energy of photon produced, $\mathbf{b}$ the photon gaussian distribution, and $\mathbf{c}$ the $x-y$ photon distribution 
mylar tape on the copper vessel hole, and (iii) $400 \mu \mathrm{m}$ of inox 316 stainless steel of the internal window of the liquid helium vessel.

The results of the simulations are shown in the two following figures relatively to electrons and a point-like electron beam source. The simulation shows an e-beam widening with a Gaussian distribution with $\mathrm{rmsx}=\mathrm{rmsy} \approx$ $240 \mu \mathrm{m}$ (Fig. 3).

The following are some comments on the simulation: (a) 200-300 microns rms are only the contribution of the cryostat window material. The simulation assumes a point source with no emittance. The spot size will be dominated by the size of the BTF beam $(1-20 \mathrm{~mm})$. The angular spread instead, 1-2 mrad, from BTF is degraded to approximately $5 \mathrm{mrad}$. In the simulation was considered also an X-ray photon production of $\sim 35 \%$ with an energy distribution below the milliequivalent and a spot size similar to the electronic distribution, with $\mathrm{rmsx}=$ rmsy $\approx 200 \mu \mathrm{m}$ (Fig. 4a-c).

The irradiation tests at the BTF have indeed to consider the X-ray contribution since the device behaves as a Josephson junction array which has already been demonstrated that are systems sensitive to X-ray radiation [17]. As a consequence, in addition, we will perform radiation tests from UV to visible and up to IR and terahertz range at the LNF. Other particles irradiation tests with conventional radiation sources are in progress or under consideration.

Acknowledgments We thank Mauro Iannarelli for the cryostat design and continuous technical support and Riccardo Ceccarelli for many useful discussions. Special thanks are due to M. Dreucci for the GEANT4 simulations.

Open Access This article is distributed under the terms of the Creative Commons Attribution 4.0 International License (http:// creativecommons.org/licenses/by/4.0/), which permits unrestricted use, distribution, and reproduction in any medium, provided you give appropriate credit to the original author(s) and the source, provide a link to the Creative Commons license, and indicate if changes were made.

\section{References}

1. Poccia, N., Baturina, T., Coneri, F., Molenaar, C.G., Wang, X.R., Bianconi, G., Brikman, A., Hilgenkamp, H., Golubov, A.A., Vinokur, V.M.: Science 349, 1202 (2015)

2. Lankhorst, M., Poccia, N.: J. Supercond. Nov. Magn. 29, 623 (2016)

3. Booth, N.E. In: Barone, A. (ed.) Superconductive Particle Detectors, Advances in Physics of Condensed Matter, p. 18 (1987)

4. Zeldov, E., Amer, N.M., Koren, G., Gupta, A.: Phys. Rev. B 39, 9712 (1989)

5. Gross, R., Marx, A., Deppe, F. In: Applied Superconductivity: Josephson Effect and Superconducting Electronics, De Gruyter Texbook (2016)

6. Santavicca, D.F., Reulet, B., Karasik, B.S., Pereverzev, S.V., Olaya, D., Gershenson, M.E., Frunzio, L., Prober, D.E.: Appl. Phys. Lett. 96, 083505 (2010)

7. Johnson, M.W., Herr, A.M., Kadin, A.M.: J. Appl. Phys. 79, 7069 (1996)

8. Hadfield, R.H.: Nat. Photonics 3, 696 (2009)

9. Cai, Y., Leath, P.L., Yu, Z.: Phys Rev. B 49, 4015 (1994)

10. Seidel, P. In: Qiu, X.G. (ed.) High-temperature superconductors, p. 324. Elsevier (2011)

11. Shapiro, S.: Phys. Rev. Lett. 11, 80 (1963)

12. Grimes, C.C., Shapiro, S.: Phys. Rev. 169, 397 (1968)

13. Zotova, A.N., Vodolazov, D.Y.: Phys. Rev. B 85, 024509 (2012)

14. Yip, S., Short, M.P.: Nature Mater. 12, 774 (2013)

15. Poccia, N., Ricci, A., Campi, G., Fratini, M., Puri, A., Di Gioacchino, D., Marcelli, A., Reynolds, M., Burghammer, M., Saini, N.L., Aeppli, G., Bianconi, A.: Proc. Nat. Acad. Sci. 109, 15685 (2012)

16. Di Gioacchino, D., Puri, A., Marcelli, A., Poccia, N., Ricci, A., Bianconi, A.: Phys. Chem. Chem. Phys. 18, 12534 (2016)

17. Rothmund, W., Zehder, A. In: Barone, A. (ed.) Superconductive particle detectors, advances in physics of condensed matter, p. 52 (1987) 